\title{
A Scanning Transmission X-Ray Microscope at the PLS: New Detection Modes
}

\author{
$\underline{\text { Hyun-Joon Shin }}^{1, *}$, Namdong Kim ${ }^{1}$ and Hee Seob Kim ${ }^{1}$. \\ 1. Synchrotron Radiation R \& D Department, Pohang Accelerator Laboratory, Pohang, Korea. \\ * Corresponding author, shj001@ postech.ac.kr
}

A scanning transmission X-ray microscope (STXM) has been operational from 2014 at the 10A beamline of the Pohang Light Source (PLS). Schematic of the STXM in normal operation mode (absorbance-change detection mode) is shown in Fig. 1(a) and a photo showing details near the sample is in Fig. 2(a). The optical components including sample stage are mounted inside a vacuum chamber $\left(10^{-5}\right.$ mbar base pressure). The incident photon flux into the STXM chamber is shown in Fig. 1(b), and practically usable photon energy range for STXM is from $\sim 150 \mathrm{eV}$ to $\sim 1600 \mathrm{eV}$. Spectral resolving power, E/ $\Delta \mathrm{E}$, of the $\mathrm{x}-$ rays is $\sim 5000$ below $1000 \mathrm{eV}$, which allows us to resolve energy level position and occupation changes, or detailed spectral changes for understanding the sample's chemical states, unoccupied electronic density of states, and/or crystal structure. As an x-ray source, $72 \mathrm{~mm}$ period elliptically polarized undulator (EPU) is used allowing us to control helicity of x-rays for understanding magnetic domain structure and dynamics by employing X-ray magnetic circular dichroism technique. Distance from the virtual source (Exit slit) to the zone plate $(\mathrm{ZP})$ is $2.5 \mathrm{~m}$. The virtual source size is typically about or less than $10 \mu \mathrm{m}$ and thus the demagnified virtual source size is generally smaller than the diffraction limited spot size; with $25 \mathrm{~nm}$ outermost zone wide $\left(\Delta r_{n}\right) Z P$, the diffraction limited resolution, $1.22 \Delta r_{n} \sim 30 \mathrm{~nm}$, is achieved upto $\sim 1000$ eV. 25, 40, and $60 \mathrm{~nm}$ ZPs are equipped for experiments. Details of normal operation mode functionality of the STXM are recently reported [1]. The STXM has been applied to transition metal doped ZnO nanoparticle catalysts [2], bio-specimen in dry condition or under wet condition [3], $\mathrm{FeO}_{\mathrm{x}}$ nanoparticle effects on bio-specimen, domain structure of magnetic thin films [4], secondary ion battery materials, and so on.

For thick samples investigation, a fluorescence measurement setup has been added as shown in Fig. 2(b). In order to secure wider collection angle, $40 \mathrm{~nm} \mathrm{ZP}$ is usually used. A single channel microchannel analyser (MCA) is placed near the sample surface. The fluorescence efficiency is low, but the sensitivity is better than the case of normal operation mode; while it was unable to locate $\mathrm{Mg}$ distribution inside a cell which was treated with $\mathrm{Mg}$ containing solution in normal mode images, $\mathrm{Mg}$ distribution inside the cell was obtained with the fluorescence measurement setup. Even though the scan speed is slow due to lower efficiency, provision of multi-elemental signals (e.g. $\mathrm{Mg}, \mathrm{O}, \mathrm{Na}$ ) at the same time is advantageous over normal operation mode. Other application examples are on understanding elemental distribution of tens of micrometer thick dust particles and elemental and valence distribution inside $\sim 3$ micrometer thick Ni-Co-Mn based secondary lithium ion battery materials. The valence state of specific element was investigated by measuring the total fluorescence yield as a function of incidence photon energy (energy scan) around the absorption edge [1].

In order to obtain better space resolution down to $10 \mathrm{~nm}$ than that of normal operation mode, Ptychography setup has been implemented as shown in Fig. 2(c). A $12.5 \mathrm{~mm}$ x $12.5 \mathrm{~mm}$ (1024 x 1024 pixels) active area CCD (Andor DX434-BN) is inserted in order to record the diffracted x-rays. The distance from the sample to the CCD surface is $\sim 8 \mathrm{~cm}$ and with this geometry the expected space resolution is estimated to be $\sim 10$ $\mathrm{nm}$ at $700 \mathrm{eV}$. Clear diffraction patterns were obtained from the $\mathrm{FeO}_{\mathrm{x}}$ clusters and from a grating pattern. 
In order to take advantage of $\mathrm{x}$-ray helicity control for XMCD investigation, magnetic field inducing setup comprised of 4 rotatable permanent magnets was installed as shown in Fig. 2 (d). By aligning the magnets, any direction of magnetic field is obtained. Maximum field in the vertical direction is 0.2 Tesla. The system has been applied for thin film domain structures [4]. In addition to this, electromagnet field application devices in the horizontal and vertical directions vs sample surface are devised.

In the presentation, the above functionalities will be provided. Also, our in-situ experimental trials will be addressed.

\section{References:}

[1] H. Shin et al., J. Synchrotron Rad. 25 (2018). http://doi.org.101107/S1600577518002564.

[2] Y. J. Hwang et al., Appl. Catalysis B: Environmental 204, 209 (2017).

[3] S. U. Yu et al., Nanoscale 10, 150 (2018).

[4] D.-T. Quach et al., Physica B 532, 221 (2018).

[5] The authors acknowledge funding from the Basic Science Research Program (NRF2015R1A5A1009962) through the National Research Foundation of Korea (NRF) funded by the Korea government Ministry of Science, ICT and Future Planning (MSIP), and appreciate Dr. Tolek Tyliszczak for many useful discussions and contributions to this work.

(a)

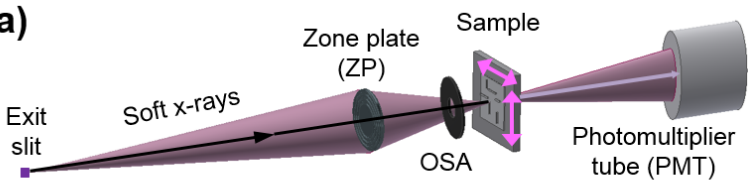

(b)

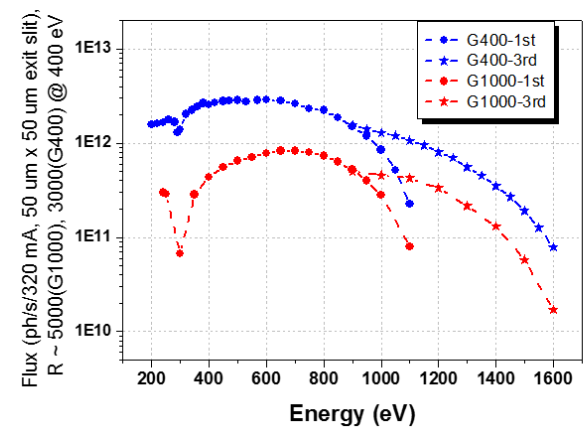

Fig. 1 (a) A schematic of a STXM in normal operation mode (transmission mode) at the 10A beamline of the PLS [1]. (b) X-ray flux measured upstream of the STXM chamber. OSA: order sorting aperture.
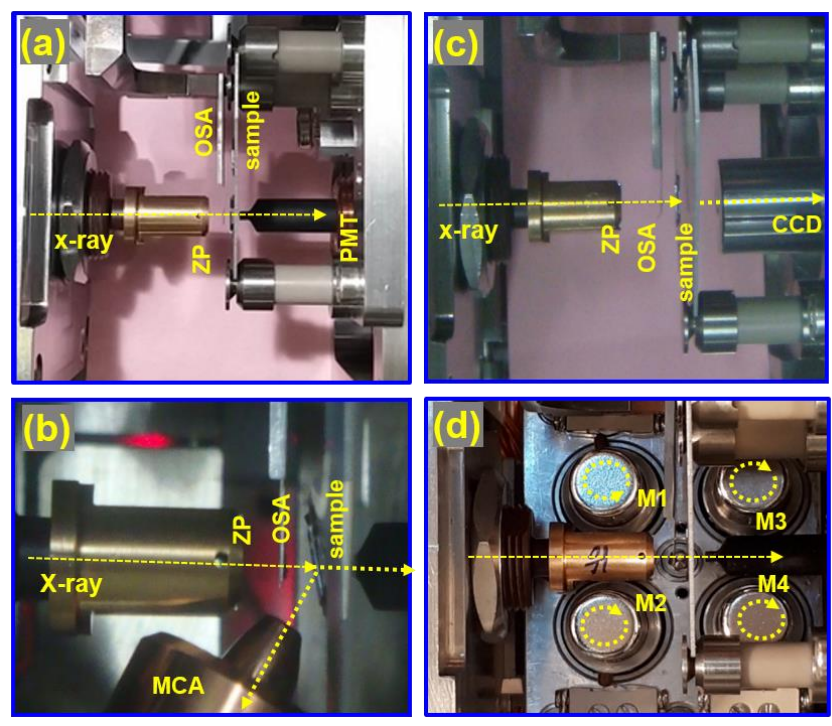

Fig. 2 Photos showing setups for (a) normal operation mode, (b) x-ray fluorescence detection mode, (c) Ptychography mode, and (d) magnetic field application mode. MCA: multichannel analyzer. 